\title{
A Laboratory Study of Iceberg Side Melting in Vertically Sheared Flows
}

\author{
A. FitzMAurice \\ Program in Atmospheric and Oceanic Sciences, Princeton University, Princeton, New Jersey \\ C. Cenedese \\ Woods Hole Oceanographic Institution, Woods Hole, Massachusetts \\ F. STRANEO \\ Scripps Institution of Oceanography, San Diego, California
}

(Manuscript received 14 September 2017, in final form 2 May 2018)

\begin{abstract}
An earlier study indicates that the side melting of icebergs subject to vertically homogeneous horizontal velocities is controlled by two distinct regimes, which depend on the melt plume behavior and produce a nonlinear dependence of side melt rate on velocity. Here, we extend this study to consider ice blocks melting in a two-layer vertically sheared flow in a laboratory setting. It is found that the use of the vertically averaged flow speed in current melt parameterizations gives an underestimate of the submarine side melt rate, in part because of the nonlinearity of the dependence of the side melt rate on flow speed but also because vertical shear in the horizontal velocity profile fundamentally changes the flow splitting around the ice block and consequently the velocity felt by the ice surface. An observational record of 90 icebergs in a Greenland fjord suggests that this effect could produce an average underestimate of iceberg side melt rates of $21 \%$.
\end{abstract}

\section{Introduction}

Icebergs discharged from the Greenland and Antarctic ice sheets constitute a combined annual mass flux of roughly $1800 \mathrm{~km}^{3}$ to the ocean (Enderlin and Howat 2014; Depoorter et al. 2013), and as such they act as a significant distributed source of freshwater as they are carried away from their source regions and melt along their trajectories. A single iceberg may also significantly influence water properties locally, by altering the ocean stratification in its vicinity and affecting the nutrient distribution through either direct release or redistribution of existing nutrients as it melts (Smith et al. 2007). In terms of the influence of iceberg melt on sea surface temperature (SST) and sea ice coverage, the literature remains undecided. Some studies suggest that icebergs act to reduce SSTs and promote sea ice growth (e.g., Merino et al. 2016), while others argue that depending on the ocean stratification this may not always be the case (Bintanja et al. 2013; Stern et al. 2017). These contradicting observations may be explained by the

\footnotetext{
Corresponding author: A. FitzMaurice, apf@princeton.edu
}

vertical distribution of iceberg meltwater, with less surface cooling and freshening observed when meltwater is well mixed in the water column (Stern et al. 2017).

For icebergs in an unstratified ocean subjected to a homogeneous flow, a recent study (FitzMaurice et al. 2017) has shown that if the velocity of the iceberg relative to the water (relative velocity hereinafter) $u$ is of greater magnitude than the vertical velocity of its melt plumes $w$, these melt plumes detach from the face of the iceberg, allowing the comparatively warm ambient fluid to be in direct contact with the iceberg. Hence, a higher submarine melt rate is observed when the melt plumes are detached compared to when they are attached to the iceberg's face. The transition, occurring when $u \approx w$, from the attached plume regime (in which the iceberg is relatively well insulated from the ambient ocean water) to the detached plume regime (where it is not) produces a highly nonlinear dependence of the side melt rate on the relative flow velocity. Further, this transition corresponds to a change in where the meltwater is distributed, from an almost undiluted surface layer of fresh meltwater in the attached plume regime to a broad region of mixed meltwater downstream of the iceberg in 

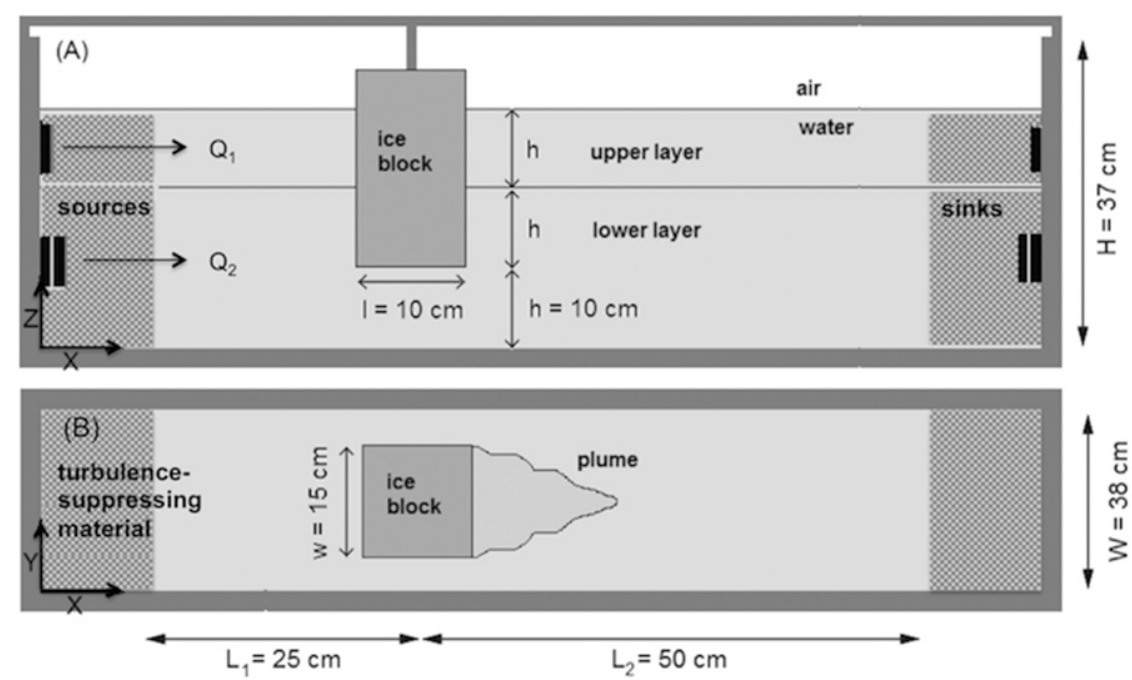

FIG. 1. A schematic (not to scale) of the laboratory setup for the vertically sheared flow experiments. (a) A side view of the ice block suspended in the working part of the flume. (b) A bird's-eye view of the ice block, with a downstream surface layer of melt plume waters.

the detached plume regime. If the meltwater is distributed in an almost undiluted surface layer, we would anticipate a reduced SST and an enhanced sea ice growth in the vicinity of icebergs, whereas if the meltwater is mixed in the water column this effect will be diminished. Thus, the contradicting effects of iceberg melt on SSTs and sea ice growth discussed above may be explained by the different melt plume behaviors, resulting from the influence of a background flow on the iceberg.

The study of FitzMaurice et al. (2017) considers icebergs melting in a uniform background flow. However, icebergs are unlikely to feel a completely homogeneous horizontal velocity profile over their draft, especially in fjord environments where strongly sheared flows are often present (Straneo et al. 2010; FitzMaurice et al. 2016). Through a series of laboratory experiments, we investigate the behavior of iceberg melt plumes in a twolayer vertically sheared flow, with implications for the modeling of iceberg submarine melt rates in the real ocean.

The structure of this manuscript is as follows. Section 2 describes the laboratory setup and methodology, section 3 outlines the experimental results, a discussion follows in section 4 , and conclusions are drawn in section 5 .

\section{Methods}

A recirculating flume (Fig. 1) of width $38 \mathrm{~cm}$ and length $200 \mathrm{~cm}$ (of which the working part was $75 \mathrm{~cm}$ long), filled with room-temperature $\left(18^{\circ}-21^{\circ} \mathrm{C}\right)$ seawater (salinity $S \approx 32 \mathrm{~g} \mathrm{~kg}^{-1}$ ), was driven by two separate pumps with flow rates $Q_{1}$ and $Q_{2}$. Pump 1 controlled the flow rate of the upper $10 \mathrm{~cm}$ of the tank, while pump 2 controlled the flow rate of the lower $20 \mathrm{~cm}$. Each pump could be individually set to one of four discrete values to give top- and bottom-layer flow speeds $u_{1}$ and $u_{2}$ of 0 , $1.5,3.3$, or $3.7 \mathrm{~cm} \mathrm{~s}^{-1}$. The difference in velocity between $u_{1}$ and $u_{2}$ generated a shear layer (observed to be approximately $5 \mathrm{~cm}$ thick) between the two layers, as is the case in the real ocean.

A fresh ice block of submerged height $20 \mathrm{~cm}$ was suspended in $30 \mathrm{~cm}$ of water, such that half of the ice was in the upper layer and half was in the lower layer (Fig. 1). The ice block was held fixed so that the flow was equivalent to that observed in the iceberg's frame of reference. Turbulence-suppressing foam and a honeycomb lattice were inserted upstream of the ice block to ensure that the flow was laminar outside of the narrow shear layer between the upper and lower layers.

The ice block was allowed to melt over a period $d T=$ $15 \mathrm{~min}$, and the resultant mass difference $d W(\mathrm{~g})$ was used to calculate a submarine melt rate $M\left(\mathrm{~cm} \mathrm{~min}^{-1}\right)$ using the formula

$$
M=\frac{d W}{\rho_{i} A_{\text {ave }} d T},
$$

where $\rho_{i}=0.92 \mathrm{~g} \mathrm{~cm}^{-3}$ is the ice density, and $A_{\text {ave }}$ is the average total submerged surface area of the ice block over the course of the experiment. To gain further understanding of where the ice melt occurs, we analyzed videos of the ice block melting to divide the total submarine melt rate into upstream, downstream, and basal 

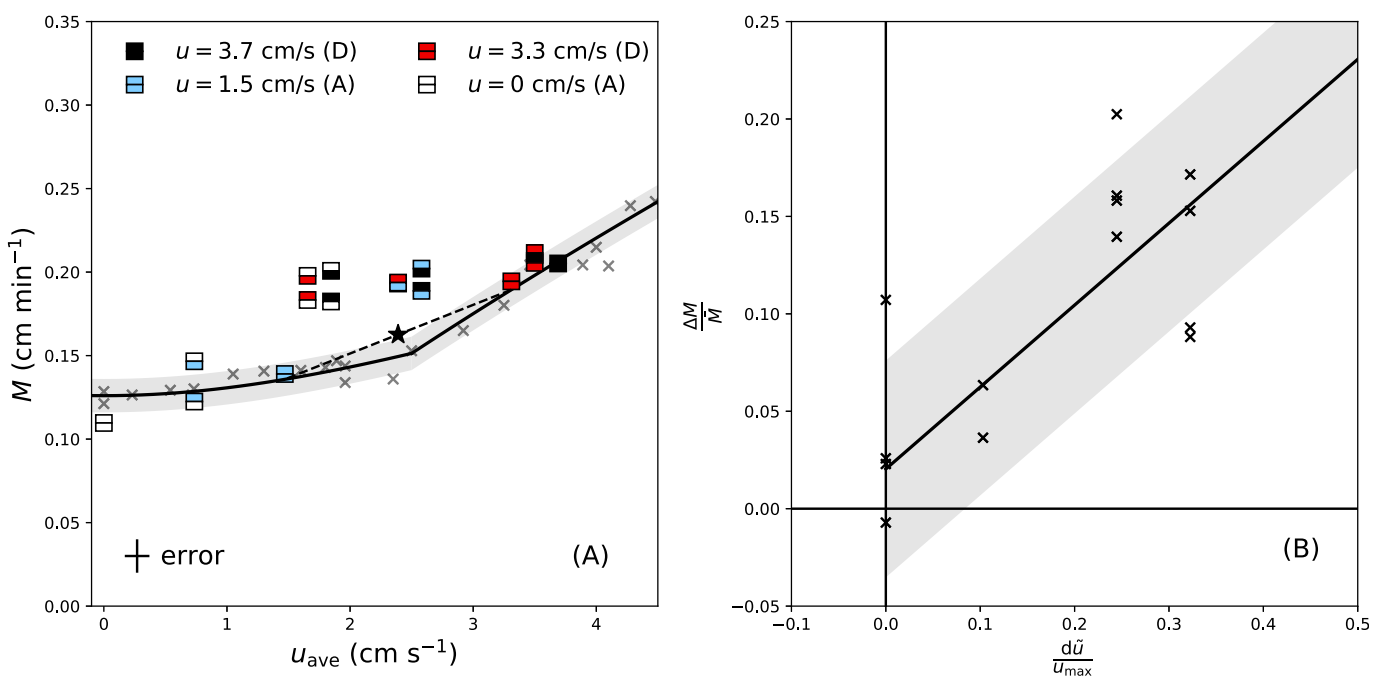

FIG. 2. (a) Measured submarine side melt rate $M$ in the vertically sheared flow experiments, overlaid on the recently proposed nonlinear parameterization for iceberg side melt in a homogenous flow (FitzMaurice et al. 2017). The coloring of the top and bottom halves of the squares indicates the top- and bottom-layer velocities in that experiment, varying from 0 (white) to $3.7 \mathrm{~cm} \mathrm{~s}^{-1}$ (black). The lettering in the legend indicates which of these velocities would ordinarily be in the attached plume regime ("A") and which would be in the detached plume regime ("D"). At $u_{\text {ave }}=3.4 \mathrm{~cm} \mathrm{~s}^{-1}$, the red-over-black square overlaps with the black-over-red square. The star is the center point of the dashed line between the side melt rates $M\left(u_{\mathrm{ave}}=1.5 \mathrm{~cm} \mathrm{~s}^{-1}\right)$ and $M\left(u_{\mathrm{ave}}=3.3 \mathrm{~cm} \mathrm{~s}^{-1}\right)$ and illustrates that $\bar{M}=(1 / 2)\left[M\left(u_{\text {ave }}=1.5 \mathrm{~cm} \mathrm{~s}^{-1}\right)+M\left(u_{\text {ave }}=3.3 \mathrm{~cm} \mathrm{~s}^{-1}\right)\right]$ is lower than the measured side melt rate $M$ (a comparable star exists for all other combinations, but this has been omitted from the figure for clarity). (b) Nondimensionalized difference $\Delta M$ between the measured submarine side melt rate and the submarine side melt rate predicted by averaging the side melt rate in each of the two layers, as a function of the nondimensionalized difference in the layer speeds $d \tilde{u}=\max \left[u_{\max }-\max \left(u_{\min }, w\right), 0\right]$. The $\Delta M$ is nondimensionalized with the experiment's average melt rate $\bar{M}$, and $d \tilde{u}$ is nondimensionalized with the experiment's maximum flow velocity $u_{\text {max }}$. In both (a) and (b), the gray shading indicates the estimated experimental error in the measured side melt rate.

components by tracking the ice edge in each of these regions (i.e., the location of the left, right, and bottom edges, respectively, in the schematics of Fig. 3; shown below). Note that this methodology only allowed the tracking of the three ice edges in the plane viewed by the video camera and requires the assumption that melting is spatially uniform in the cross-flow direction (observations of the melted ice block at the end of the experiments confirmed that this is a good assumption, at leading order). The contribution of basal melting to the total melt rate, as calculated from the videos, was removed prior to the analysis to isolate the side submarine melt rate, which is the focus of this study.

\section{Results}

The recent study of FitzMaurice et al. (2017) showed that, because of the existence of two distinct regimes (i.e., sideattached and side-detached melt plumes for low and high background flows, respectively), the side melt rate of icebergs has a highly nonlinear dependence on the upstream horizontal flow velocity (black curve in Fig. 2a). It follows that for an iceberg subject to a vertically sheared flow in which different regimes (side-attached and side-detached) occur over its draft, the side melt rate calculated using the vertically averaged horizontal flow velocity in this nonlinear parameterization will be less than the side melt rate calculated by applying the parameterization at each depth and subsequently averaging. Mathematically, this may be expressed (for an iceberg of draft $D$ ) as follows:

$\bar{M}=\frac{1}{D} \int_{-D}^{0} M[u(z)] d z>M\left[\frac{1}{D} \int_{-D}^{0} u(z) d z\right]=M(\bar{u})$

For a two-layer vertically sheared flow, the anticipated average side melt rate $\bar{M}$ will lie at the center of the straight line drawn between the submarine side melt rates $M\left(u_{1}\right)$ and $M\left(u_{2}\right)$ in each of the two layers (e.g., dashed line and star in Fig. 2a). However, the laboratory results show that the measured side melt rate in a two-layer vertically sheared flow exceeds even this $\bar{M}$ (Fig. 2a). Note that allowing for a side melt rate that continuously varies with velocity across the interfacial shear layer (which was calculated to be approximately $5 \mathrm{~cm}$ thick) does not significantly alter this result. 


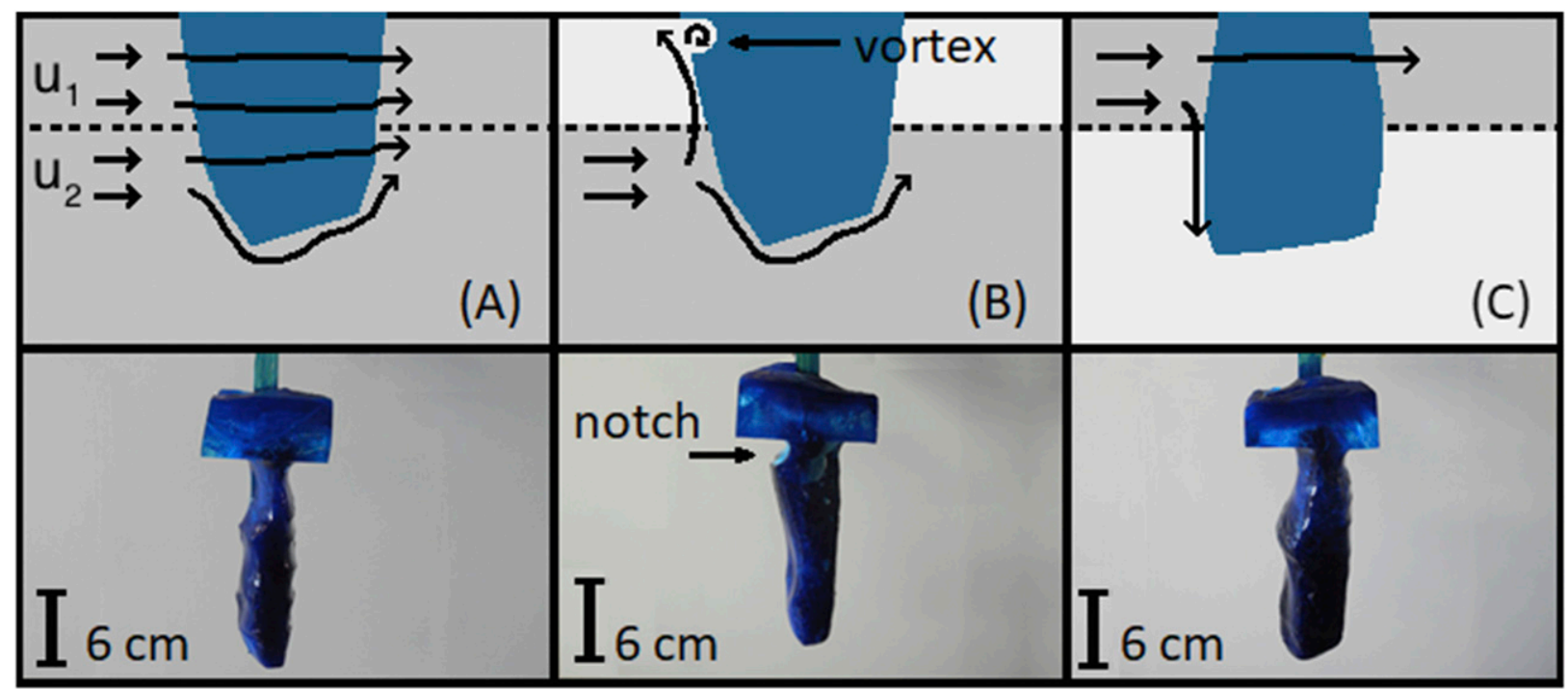

FIG. 3. (top) Schematic of an ice block in a two-layer vertically sheared flow in which (a) $u_{1}=u_{2}$, (b) $u_{1}<u_{2}$, and (c) $u_{1}>u_{2}$. In (a), the flow is predominantly horizontally around the ice block, whereas in (b) and (c) it is deflected up and down, respectively, into the lower velocity layer. (bottom) Photos of the ice block after $15 \mathrm{~min}$ of melting for (a) $u_{1}=u_{2}=3.3 \mathrm{~cm} \mathrm{~s}^{-1}$, (b) $u_{1}=0, u_{2}=3.3 \mathrm{~cm} \mathrm{~s}^{-1}$, and (c) $u_{1}=3.3, u_{2}=0 \mathrm{~cm} \mathrm{~s}^{-1}$.

The reason for this excess in side melt rate may be understood by considering a phenomenon that was observed to occur when there was no or a low horizontal velocity in the top layer of the flume and a high horizontal velocity in the bottom layer of the flume (i.e., in the cases where $u_{2}>u_{1}$ ). In these experiments, a small semicircular notch of vertical diameter $2-3 \mathrm{~cm}$ formed below the waterline across the horizontal extent of the upstream face of the ice block (Fig. 3b). The formation of this notch was associated with a rapidly rotating clockwise vortex located against the ice face at this level (Fig. 3b), which was directly observed by depositing potassium permanganate and colored dye in the flow (not shown). It was found that this vortex was a feature generated by the sheared flow encountering an obstacle and formed near the free surface even when a wooden block (issuing no buoyancy and no meltwater plume) of the same dimensions as the ice block was positioned in the flume in place of the ice block. The vortex was generated by the lower-layer flow being diverted preferentially upward, instead of solely horizontally around the block, when the upper layer was stationary (Fig. 3b). When this vertical flow along the upstream face of the block encountered the free surface, it was deflected away from the block and developed a vortex (rotating in a sense counter to that of the primary flow) at the corner between the free surface and the block, which is a previously observed feature of a flow around a corner (e.g., Erturk 2009).

In the reverse scenario, with a stationary lower layer and a fast-moving upper layer, the flow was likewise observed to be preferentially deflected vertically (this time downward) into the stationary layer. However, the absence of a free surface at the base of the ice block prevented the formation of a basal notch similar to the surface notch observed in the vertically reversed case. The three different scenarios (homogeneous flow, faster lower layer, and faster upper layer) are illustrated schematically in Fig. 3. Note that the mass lost as a result of the formation of a notch, in the case of slow flow overlying a fast flow, accounts for the higher side melt rates observed in these experiments relative to the equivalent fast flow overlying slow flow experiments; that is, the white-topped square symbols in Fig. 2a have larger submarine side melt rate than the white-bottomed symbols with the same average flow velocity.

This tendency for the flow to deflect vertically (from a fast-flowing layer into a slow-flowing layer) as opposed to horizontally around the ice block when there is vertical shear in the horizontal flow is key to understanding the enhanced melt rates observed in sheared flows. The presence of a high horizontal velocity in one layer induces the ice surface to experience an enhanced vertical velocity in any more slowly flowing layers, and this raises the mean side melt rate above that predicted by averaging the side melt rates corresponding to each of the upstream layer velocities. This deflection effect may be quantified using the metric $d \tilde{u}=\max \left[u_{\max }-\max \left(u_{\min }, w\right), 0\right]$, which is the difference between the fast flow layer speed $u_{\max }$ and the effective speed in the slow flow layer (i.e., the maximum of the slow layer horizontal speed $u_{\min }$ and the vertical 
plume speed $w$ ) when this value is positive, and zero otherwise. Here, the vertical plume speed was estimated to be of order $2.5 \mathrm{~cm} \mathrm{~s}^{-1}$ via feature tracking of the dyed meltwater in videos of the ice melting, and more precisely inferred from the transition between the attached and detached plume regimes occurring when the barotropic horizontal background flow speed is equal to $2.5 \mathrm{~cm} \mathrm{~s}^{-1}$ (FitzMaurice et al. 2017). Defining $\Delta M=M-\bar{M}$, where $M$ is the observed submarine side melt rate and $\bar{M}=(1 / 2)\left[M\left(u_{\mathrm{ave}}=u_{1}\right)+M\left(u_{\mathrm{ave}}=u_{2}\right)\right]$, it is found that this difference between the observed and predicted melt rate $\Delta M$ increases linearly as a function of $d \tilde{u}$, the measure of the effect of flow deflection around the ice block (Fig. 2b). The above quantities have been nondimensionalized with the experiment's average melt rate $\bar{M}$ and the experiment's maximum flow velocity $u_{\text {max }}$, respectively.

\section{Discussion}

Laboratory results suggest that the way ocean currents are deflected around icebergs may have a significant impact on the iceberg side melt rate, via the relative ice-ocean velocity. This effect is likely to be not just a function of the vertical shear in the horizontal velocity profile but also of the iceberg aspect ratio and the particulars of its subsurface geometry. For instance, it might be anticipated that for icebergs with a cross-flow horizontal scale that is large relative to their depth, the flow will be preferentially diverted under the ice as opposed to horizontally around it. This could enhance the vertical velocity felt by the upstream ice face or, if the downward flow slows a surface-attached upstream melt plume, decrease it. Such a scenario would be of particular relevance to Antarctic icebergs, which generally have a large aspect ratio compared to their Greenlandic counterparts.

Observations of icebergs in a fjord environment suggest that icebergs move predominantly with the vertical average of the ocean currents (FitzMaurice et al. 2016). Consequently, the flows used to simulate relative iceberg-ocean velocities in the present study are unrealistic in that they do not integrate to zero over the iceberg draft. However, it is reasonable to assume that the directionality of the flow will only have a secondorder impact on the ice block melt rate, and as such the flows studied here may be considered analogous to flows in which the layers move at equivalent speeds but in opposite directions, such that the iceberg motion is given by the integrated currents over its draft. The case of a two-layer shear flow, with velocities in opposite directions and of very different magnitudes in each layer, can occur such that the horizontal relative ice-ocean velocity integrates to zero over the iceberg's draft, provided that the extent of the iceberg in each of the two layers is different (e.g., FitzMaurice et al. 2016; Figs. 3b,c). In this case, we would expect flow deflection to occur and similar dynamics to be observed in the ocean as in the laboratory. A scenario in which there is a relative icebergocean velocity of uniform direction could, of course, be sustained for a grounded iceberg or for an iceberg not moving with the vertical average ocean currents because of, for example, strong wind forcing.

The shear layer between the upper and lower velocity layers in the present experiments was observed to be approximately $5 \mathrm{~cm}$ thick, covering a quarter of the ice block draft of $20 \mathrm{~cm}$. The vertical ocean velocity profiles shown in Fig. 3 of FitzMaurice et al. (2016) show a typical shear layer thickness of order $50-100 \mathrm{~m}$ in Sermilik Fjord at times during which vertical shear was present in the ocean currents. The draft range of icebergs passing the sensor in that study was 7 to $490 \mathrm{~m}$. Thus, the fraction of the iceberg draft influenced by the shear layer is comparable to that obtained in the laboratory experiments. This is especially true for the largest icebergs in the fjord, which contribute the largest melt flux to the ocean.

The metric $d \tilde{u}$, quantifying the flow deflection around the ice block, when appropriately nondimensionalized with the maximum background flow speed, can be calculated for the observed relative velocity profiles experienced by icebergs in a Greenlandic fjord in FitzMaurice et al. (2016). The shear profiles of these oceanic flows are not entirely analogous to the two-layer laboratory setup, but by defining the oceanic $d \tilde{u}$ using the maximum and minimum relative ice-ocean velocities over the iceberg draft we can make an approximate estimate of the error incurred in parameterizing the melt rates of icebergs in sheared ocean flows. Conducting this analysis, it is found that the average oceanic $d \tilde{u} / u_{\max }=0.48$, suggesting an average underestimate of the parameterized side melt rate by $21 \%$. Over the full observational dataset, the oceanic $d \tilde{u} / u_{\max }$ varied between 0 and 0.86 , resulting in the parameterized meltrate error ranging between $0 \%$ and $38 \%$. The laboratory experiments, with $d \tilde{u}$ varying between 0 and 0.32 , thus covered a significant range of the oceanic values of this parameter. It is anticipated that the oceanic $d \tilde{u}$ will be largest in highly sheared fjord environments. However, in the open ocean icebergs may be deep enough relative to the Ekman layer to experience sufficient vertical shear that the assumptions made by current models might not hold.

The magnitude of the melt-rate enhancement due to flow deflection seen in this study is anticipated to be dependent on the height reached by the flow when it is 
deflected vertically by the upstream face of the ice block, which may be reduced by the stratification in the fjord. However, the deflected flow will be mixed with the buoyant water of the melt plume, so we anticipate that the upper bound on the scale height reached by the deflected flow will be the plume's level of neutral buoyancy. There is observational evidence of iceberg melt plumes reaching the ocean surface (e.g., Yankovsky and Yashayaev 2014), and it is thus reasonable to assume that flow deflection effects are important on scales relevant to icebergs in the ocean.

The laboratory experiments described in this manuscript were designed to conduct a process study of the dynamical impact of a sheared flow on the side melting of an ice block. Since the dominant mechanism controlling the difference in melt rates between the different experiments is the flow dynamics (i.e., the plume regimes and the flow deflection), we do not anticipate that the ice thermodynamics play a fundamental role in this study. Hence, we expect that if the same experiments are conducted at a lower, more realistic temperature, the flow dynamics will remain unchanged.

A change in temperature will enter this parameterization via a change in the heat transfer coefficient (through changes to the Prandtl number and the thermal conductivity of water) and via a change in the plume vertical velocity $w$, which depends on the plume buoyancy flux. At lower temperatures, $w$ is expected to decrease, owing to the reduced buoyancy flux associated with a lower melt rate, and consequently the transition from the attached to the detached plume regime will occur for lower relative velocities. Comparison with observational data has been conducted using realistic fjord temperatures in the parameterization of FitzMaurice et al. (2017), for a plume vertical speed of $0.05 \mathrm{~m} \mathrm{~s}^{-1}$, following the modeling of Sciascia et al. (2013) and consistent with iceberg melt plume observations (Josberger and Neshyba 1980). This comparison took account of both the different temperature (via the plume vertical velocity, the Prandtl number, and the thermal conductivity) and the different Reynolds number (which enters via the Nusselt number) in oceanic conditions and found that the laboratory-derived parameterization predicts melt rates that are comparable to those that have been observed for icebergs in polar ocean waters (e.g., Enderlin and Hamilton 2014). So while this study alone may not give a complete picture of iceberg melt rates, its limitations are not a first-order problem, and we believe it is a necessary step in understanding how icebergs melt in different oceanic flows.

\section{Conclusions}

In the presence of an ambient vertically sheared flow, using the vertically averaged horizontal velocity in the recently proposed nonlinear iceberg side melt parameterization (FitzMaurice et al. 2017) results in an underestimate of iceberg side melting, particularly when the shear is large. In part, this follows from the nonlinearity of the dependence of side melt on relative velocity if the velocity range spans both the attached and detached regimes [Eq. (2)]. However, another important contribution is the fact that the iceberg acts as an obstacle to the flow, and as such the horizontal velocity in one layer may influence the flow in a different layer. Hence, even if nonlinearity is taken into consideration by calculating the submarine side melt rate separately in each different layer and subsequently averaging the side melt rates [Eq. (2)], the total submarine side melt rate is still underestimated (Fig. 2a). In particular, if there is a fast flow in one layer and a slow flow in the other layer, the flow around the ice block ceases to be approximately horizontal and instead is deflected vertically from the high-velocity layer to the low-velocity layer.

The difference between the observed submarine side melt rate and the average of the predicted submarine side melt rates in each of the two layers is found to be a linearly increasing function of a metric of the velocity difference between the layers. Qualitatively, then, the error introduced by not accounting for the flow deflection around an iceberg is likely to be small when the horizontal velocity profile is relatively homogeneous with depth and large when there is a strong vertical shear. By nondimensionalizing the shear metric with the maximum background flow speed and applying this relationship to observed relative ice-ocean velocity profiles (FitzMaurice et al. 2016), it was estimated that neglecting flow deflection effects will cause iceberg side melt rates to be underestimated by $21 \%$, on average. Further studies of flow past an obstacle are required to find the precise value of the correction to the side melt rate that is necessary because of the flow deflection, as it is anticipated that the specifics of this relationship will be a function not just of the vertical shear in the horizontal velocity but also of the ice block aspect ratio and geometry. The influence of this flow deflection may be important in an ocean setting (particularly in environments with large vertical shear, such as Greenlandic fjords) but will not be resolved by ocean models that do not explicitly model the flow around icebergs. It is thus important to bear in mind that iceberg melt parameterizations likely underestimate iceberg melt rates in such scenarios.

Acknowledgments. The authors thank A. Jensen for his assistance in the laboratory and B. Sutherland for his insightful comments. A. F. was supported by NA14OAR4320106 from the National Oceanic and 
Atmospheric Administration, U.S. Department of Commerce. The statements, findings, conclusions, and recommendations are those of the authors and do not necessarily reflect the views of the National Oceanic and Atmospheric Administration or the U.S. Department of Commerce. C. C. was supported by NSF OCE-1658079 and F. S. was supported by NSF OCE-1657601 and NSF PLR-1743693.

\section{REFERENCES}

Bintanja, R., G. J. van Oldenborgh, S. S. Drijfhout, B. Wouters, and C. A. Katsman, 2013: Important role for ocean warming and increased ice-shelf melt in Antarctic sea-ice expansion. Nat. Geosci., 6, 376-379, https://doi.org/10.1038/ngeo1767.

Depoorter, M. A., J. L. Bamber, J. A. Griggs, J. T. M. Lenaerts, S. R. M. Ligtenberg, M. R. van den Broeke, and G. Moholdt, 2013: Calving fluxes and basal melt rates of Antarctic ice shelves. Nature, 502, 89-92, https://doi.org/10.1038/nature12567.

Enderlin, E., and G. S. Hamilton, 2014: Estimates of iceberg submarine melting from high-resolution digital elevation models: Application to Sermilik Fjord, East Greenland. J. Glaciol., 60, 1084-1092, https://doi.org/10.3189/2014JoG14J085.

- , and I. Howat, 2014: An improved mass budget for the Greenland Ice Sheet. Geophys. Res. Lett., 41, 866-872, https:// doi.org/10.1002/2013GL059010.

Erturk, E., 2009: Discussions on driven cavity flow. Int. J. Numer Methods Fluids, 60, 275-294, https://doi.org/10.1002/fld.1887.

FitzMaurice, A., F. Straneo, C. Cenedese, and M. Andres, 2016: Effect of a sheared flow on iceberg motion and melting. Geophys. Res. Lett., 43, 12 520-12 527, https://doi.org/10.1002/2016GL071602.
, C. Cenedese, and F. Straneo, 2017: Nonlinear response of iceberg side melting to ocean currents. Geophys. Res. Lett., $\mathbf{4 4 ,}$ 5637-5644, https://doi.org/10.1002/2017GL073585.

Josberger, E. G., and S. Neshyba, 1980: Iceberg melt-driven convection inferred from field measurements of temperature. Ann. Glaciol., 1, 113-117, https://doi.org/10.3189/ S0260305500017080.

Merino, I., J. Le Sommer, G. Durand, N. C. Jourdain, G. Madec, P. Mathiot, and J. Tournadre, 2016: Antarctic icebergs melt over the Southern Ocean: Climatology and impact on sea ice. Ocean Modell., 104, 99-110, https://doi.org/10.1016/ j.ocemod.2016.05.001.

Sciascia, R., F. Straneo, C. Cenedese, and P. Heimbach, 2013: Seasonal variability of submarine melt rate and circulation in an East Greenland fjord. J. Geophys. Res. Oceans, 118, 24922506, https://doi.org/10.1002/jgrc.20142.

Smith, K. L., B. H. Robison, J. J. Helly, R. S. Kaufmann, H. A. Ruhl, T. J. Shaw, B. S. Twining, and M. Vernet, 2007: Free-drifting icebergs: Hot spots of chemical and biological enrichment in the Weddell Sea. Science, 317, 478-482, https://doi.org/10.1126/ science. 1142834 .

Stern, A. A., A. Adcroft, O. Sergienko, and G. Marques, 2017: Modeling tabular icebergs submerged in the ocean. J. Adv. Model. Earth Syst., 6, 513-526, https://doi.org/10.1002/ 2017MS001002.

Straneo, F., G. S. Hamilton, D. A. Sutherland, L. A. Stearns, F. Davidson, M. O. Hammill, G. B. Stenson, and A. RosingAsvid, 2010: Rapid circulation of warm subtropical waters in a major glacial fjord in east Greenland. Nat. Geosci., 3, 182-186, https://doi.org/10.1038/ngeo764.

Yankovsky, A. E., and I. Yashayaev, 2014: Surface buoyant plumes from melting icebergs in the Labrador Sea. Deep-Sea Res. I, 91, 1-9, https://doi.org/10.1016/j.dsr.2014.05.014. 